\title{
In Response to Letter to the Editor: Torsney KM, Cocker DM, Slesser AAP (2015) The Modern Surgeon and Competency Assessment: Are the Workplace-Based Assessments Evidence- Based?
}

\author{
Kelli Torsney $^{1} \cdot$ Daniel Cocker $^{2} \cdot$ Alistair Slesser $^{3}$
}

Published online: 21 August 2015

(c) Société Internationale de Chirurgie 2015

We agree there is no evidence for extreme numbers of workplace-based assessments (WBAs), and we do not dispute that when conducted in a formative capacity, they can supplement training. However, WBAs were introduced to postgraduate training amongst the UKs medical fraternities primarily as a formative tool to promote continuous learning and yet are now being used as a summative tool to demonstrate competency. In our systematic review, only five studies looked at WBAs in a surgical setting with none of these studies investigating WBAs as a summative tool.

Indeed the situation may now be worse, with the Joint Committee on Surgical Training (JCST) issuing their recommendations for the award of a certificate of completion of training (CCT) in General Surgery. In this document, it has been recommending that trainees approaching the end of training require Procedure-Based Assessments signed off at Level 4 , competent to perform the procedure unsupervised (could deal with complications that arose), by three assessors. This is a direct summative use of these assessments, and in direct contrast to the reasons that they were introduced.

Van der Vleuten [1] demonstrated that reliability, validity, educational impact, acceptability, and cost were important considerations when devising an assessment tool. In the presented work, cost was replaced by feasibility, as the trainees do not have to pay directly for their

Kelli Torsney

kellitors@gmail.com

1 Emergency Department, West Middlesex Hospital, Isleworth, UK

2 Surgery, Watford General Hospital, Watford, UK

3 Surgery, Chelsea and Westminster Hospital, London, UK use of the WBAs, and the only hindrance to the completion of assessments is the feasibility of the assessment's completion in terms of time in busy clinical practice.

The letter that you write draws our attention to the acceptability, and this is an aspect that is as important as the others, but is the subject of precious little research. Research investigating such an area might help understand why these assessments can be so unpopular but will not necessarily improve the acceptability. Moreover, herd mentality does ensure that trainees are more or less likely to complain dependent upon the attitudes of peers.

In order to improve the acceptability of these assessments in UK postgraduate medical and surgical training, evidence of the educational impact and a cessation in the use of these assessments for summative purposes are needed quickly.

\section{Reference}

1. Van der Vleuten C (1996) The assessment of professional competence: developments, research and practical implications. Adv Health Sc Educ 1:41-67 